Fecha de recepción: septiembre 2019 Fecha de aceptación: noviembre 2019 Versión final: febrero 2020

\section{El diseño y el trabajo interdisciplinario en la investigación y gestión de contenidos patrimoniales}

\author{
Sofía Martínez Larraín ${ }^{(1)}$ y
}

Lía Valenzuela Echenique ${ }^{(2)}$

Resumen: El presente artículo describe cómo, a través de un trabajo interdisciplinario entre el diseño, la historia y el uso de recursos tecnológicos, se logra desarrollar en el año 2018 un proyecto de investigación, rescate, registro y difusión patrimonial.

El objetivo principal fue poner en valor la vida y obra del desconocido pintor chileno de fines del siglo XIX Manuel Aspillaga Valenzuela (1870-1930), para luego difundir los resultados a través de recursos digitales situados en una plataforma online de acceso gratuito. En términos de contenido el proyecto logra situar al artista y su obra dentro del contexto histórico nacional. Junto con lo anterior, el proyecto busca dar acceso a toda la comunidad a estas obras patrimoniales desconocidas a través de la tecnología y el diseño. Resulta relevante, además, la descripción de la metodología de trabajo interdisciplinario utilizada para la concreción de los objetivos. Con dicha metodología se logró un trabajo ordenado y profundo cuyo éxito se basó en potenciar cada una de las disciplinas involucradas en la investigación, ejecución, análisis y creación de piezas. El proyecto se desarrolló en tres etapas, investigar la vida y obra pictórica de Manuel Aspillaga; identificar, registrar y catalogar las obras del artista; y, por último, difundir la figura y obra de Manuel Aspillaga Valenzuela.

Esta investigación se realizó en la ciudad de Santiago de Chile en un plazo de un año.

Palabras clave: diseño - interdisciplina - patrimonio - arte - tecnología.

[Resúmenes en inglés y portugués en las páginas 315-316]

(1) Diseñadora Pontificia Universidad Católica de Chile. Máster en Historia y Gestión del Patrimonio Universidad de los Andes, Chile. Profesor de la Facultad de Diseño Universidad del Desarrollo.

(2) Historiadora Pontificia Universidad Católica de Chile. Máster en Historia y Gestión del Patrimonio Universidad de los Andes, Chile. 


\section{Introducción}

El presente artículo nace con el interés de evidenciar el trabajo interdisciplinario entre el diseño y la historia al servicio de la investigación y rescate patrimonial. Intentamos romper la creencia de que son disciplinas desvinculadas, ya que, si bien la primera se enfoca en la creatividad y la invocación, la segunda podría correr el riesgo de caer en lo obsoleto y antiguo. Sin embargo, en este proyecto se produce una integración interdisciplinaria tal y como la entiende Piaget (1979): "La cooperación entre disciplinas conlleva interacciones reales, es decir, una verdadera reciprocidad en los intercambios y, por consiguiente, un enriquecimiento mutuo. En consecuencia, llega a lograrse una transformación de los conceptos, las metodologías de investigación y de enseñanza”. Lo anterior se demostrará en la descripción de las actividades del proyecto. En este sentido, resulta valioso además mostrar la utilidad y efectividad del trabajo conjunto a partir de una metodología propia aplicada sobre esta investigación de la vida y obra del artista Manuel Aspillaga, de la que no existía registro a excepción de una breve reseña y una obra pictórica en el sitio web de artistas visuales chilenos del Museo Nacional de Bellas Artes ${ }^{1}$.

\section{Materiales y método}

Para cumplir con el objetivo general de la investigación de poner en valor la figura y obra del pintor Manuel Aspillaga Valenzuela se determinaron tres objetivos específicos: investigación de su vida; catalogación y análisis crítico de la obra; y difusión de su figura y obra. Cada objetivo específico generó los siguientes productos asociados a actividades:

1. Se escribió una biografía oficial a partir de la investigación bibliográfica de la vida de Manuel Aspillaga y su creación artística situándola en su contexto nacional.

2. Se entrevistó a desendientes directos poseedores de cuadros (19 familiares en total).

3. Se investigó en los registros de alumnos de la Facultad de Derecho y la Dirección de Asuntos Estudiantiles de la Universidad de Chile, con el fin de encontrar información sobre sus años de estudio en dicho establecimiento.

4. Se revisó la carpeta del artista en el archivo del Museo Nacional de Bellas Artes y se recopilaron datos de su época con bibliografía, principalmente, de historiadores chilenos. 5. Posterior al análisis bibliográfico, se realizó un trabajo de catalogación de las obras, lo que incluyó un registro fotográfico de ellas realizado en las casas de los descendientes propietarios. Junto a estas, se realizó la catalogación de una obra ubicada en el Museo de Bellas Artes (50 obras en total).

6. Una vez identificadas y catalogadas las obras, se realizó un análisis crítico de estas, tomando en cuenta los datos biográficos, las obras y su iconografía e información sobre artistas del período que pudieron haber influido en Aspillaga.

7. Como resultado de lo anterior, se diseñó un catálogo gráfico y visual de las obras encontradas del artista donde, junto con la imagen de cada una, se detallan sus dimensiones, estado de conservación, su fisonomía, entre otros datos de interés. 
8. Para la obtención de las imágenes se tomaron fotografías profesionales de cada pintura con equipo de iluminación y cámara digital.

9. Por último y con el objetivo de dar a conocer al pintor y su obra catalogada se diseñó un sitio web bajo el dominio www.manuelaspillaga.com que contiene toda la información e imágenes recopiladas durante la investigación, con el fin de dar acceso a toda la comunidad interesada en la información.

10. Dentro del sitio web el usuario puede acceder visualmente a toda la obra como si estuviera en un museo y también puede descargar el catálogo digital que contiene el contexto histórico, la biografía y obra del artista en un solo documento de atractivo gráfico.

El sitio web se constituyó como un gran aporte a la difusión del proyecto ya que este se encontrará habilitado de manera gratuita, pudiendo no solo acceder a la obra y la investigación, sino también contactarse con las creadoras en caso de poseer más información que de manera colaborativa complemente la investigación o bien solicitar otros datos de interés.

\section{Cronología del proyecto}

\section{Objetivo específico $\mathrm{n}^{\circ} 1$ : Investigar la vida y obra pictórica de Manuel Aspillaga}

\section{Biografía}

Luego del inicio de proyecto de parte de las alumnas, se comenzó estableciendo los primeros contactos con Hernán Rodríguez Villegas, director del Museo Andino (quien propuso el presente trabajo a la universidad). Él entregó un acercamiento sobre la familia, una investigación preliminar y un árbol genealógico del artista, sobre el que se comenzó la investigación. A partir de lo anterior, se planificó la investigación de la siguiente manera: Durante los meses de abril a julio se realizó una investigación bibliográfica a partir de diversas fuentes de información:

- Visita a la Facultad de Derecho y la Dirección de Asuntos Estudiantiles de la Universidad de Chile con el fin de encontrar información sobre sus años de estudio en dicho establecimiento.

- Visita al archivo del Museo Nacional de Bellas Artes.

- Recopilación de bibliografía a partir, principalmente, de historiadores chilenos.

Los meses de agosto y noviembre se complementó la investigación anterior con información obtenida a partir de las siguientes fuentes:

- Reunión con 19 miembros de la familia Aspillaga, descendientes del artista.

- Transcripción de 11 entrevistas realizadas a descendientes de Manuel Aspillaga sobre el artista. 
- Recopilación de documentos otorgados por la familia Aspillaga en base a investigaciones familiares sobre el artista.

- Objetos otorgados por la familia en las visitas a las casas.

Finalmente, durante el mes de noviembre, se procedió a redactar una biografía (incluida en la tercera parte del presente trabajo). Esta enumera todas las fuentes mencionadas con anterioridad. Una vez completada esta primera parte la profesora guía Isabel Cruz Amenábar, historiadora del arte, realizó una primera revisión del avance. Una vez hechas las modificaciones e incorporando nuevos antecedentes, se realizó una segunda recorrección, para finalmente ser entregada en su formato definitivo y alcanzando lo propuesto a inicios del proyecto.

\section{Análisis crítico de la obra}

Luego de un primer encuentro con Hernán Rodríguez, y gracias a su información y referencias de contactos con la familia, se estableció un vínculo con Gonzalo Aspillaga y Soledad Vergara de Aspillaga, quienes durante el mes de abril se reunieron con las investigadoras para enseñarnos un pequeño álbum con una recopilación de las obras de su abuelo, que sirvieron para dimensionar su trabajo y calidad.

Con la información obtenida de este precario registro se realizó un catastro de las obras artísticas, durante los meses de abril a junio. Luego se estableció contacto con las cuatro ramas descendientes de Manuel Aspillaga y a su vez con todos sus nietos, entre los meses de agosto y noviembre, con el fin de obtener un informe exacto del número de obras y su ubicación. De este modo, se elaboró un documento de planificación con el detalle de cada familia y sus posesiones relacionadas al artista, números de contacto y posible ubicación geográfica de las obras.

Entre los meses de agosto y noviembre, se fotografiaron y catalogaron 50 cuadros en formato profesional visitando en sus casas a los respectivos dueños. Estos cuadros fueron impresos y ordenados cronológicamente en un álbum de fotografías con el fin de realizar un análisis adecuado de las obras. Se establecieron líneas generales sobre el estilo del artista y algunas primeras hipótesis que fueron conversadas con la profesora guía a inicios de noviembre.

Luego de la redacción del análisis artístico, durante el mes de noviembre, la profesora guía realizó una corrección que permitió perfeccionar y corregir el documento. Después de una segunda recorrección, se hizo entrega del documento definitivo que se estipuló como meta durante la gestión del proyecto.

\section{Objetivo específico $n^{\circ} 2$ : Identificar y catalogar las obras del artista}

\section{Catalogación de las obras}

Con el fin de realizar un catálogo de las obras del artista se procedió, en primer lugar, a elaborar una ficha tipo con 17 ítems en base a las fichas propuestas por la profesora Lina Nagel en su libro Manual de Registro y Documentación de Bienes Culturales que fueron 
posteriormente corregidos por la profesora guía Isabel Cruz, durante el mes de agosto. La catalogación se planificó de la siguiente manera entre agosto y noviembre:

- Visita de 20 residencias particulares, con el fin de catalogar las obras.

- Catalogación por medio de fichas prestablecidas en terreno de 50 obras artísticas de Manuel Aspillaga.

- Corrección de las 50 fichas en base a un formato y vocabulario común.

Finalmente, se estableció un formato definitivo de fichas, el que fue corregido por la profesora guía, distinguiendo entre las fichas difusivas y las familiares (poseen datos privados como dirección, propietario, año de adquisición e historia de la obra en cuestión).

Durante el mes de diciembre, la profesora guía corrigió la totalidad de las fichas, las que se encontraban en su formato definitivo, lográndose con esto llegar a la meta propuesta en los inicios del proyecto.

Posteriormente, se diseñó un catálogo digital con todo el material mencionado. A continuación, un ejemplo de las páginas ya diseñadas en base a un orden de ficha que se estableció en conjunto con la profesora guía Isabel Cruz, que consta de 17 puntos en el siguiente orden:

1. Título de la obra

2. Autor

3. Datación o época

4. Firmas

5. Dimensiones

6. Técnica/Material

7. Descripción física (incluye análisis de técnica pictórica y paleta cromática, estilo gráfico y formato)

8. Breve historia de la obra

9. Estado de conservación

10. No de inventario

11. Ubicación actual

12. Fecha de adquisición

13. Propietarios

14. Objeto

15. Clasificación

16. Observaciones

17. Fichado por

\section{Fotografías profesionales}

Para poder realizar el presente producto se trabajó de la siguiente manera entre abril y junio:

- Se realizó un catastro preliminar sobre las obras existentes del artista.

- Se establecieron los primeros contactos con algunos miembros de la familia Aspillaga. 
- Se realizó la compra de equipo profesional de iluminación que consistió en tres focos LED de iluminación con difusor con sus respectivos trípodes.

- Se utilizó una cámara digital Nikon D7000 y, en algunas ocasiones, se recurrió a un iPad Pro Retina por las condiciones de espacio del lugar.

Los meses de agosto y noviembre se llevaron a cabo las siguientes acciones:

- Visita de 20 domicilios particulares que poseían cuadros.

- Fotografías profesionales de 50 cuadros del artista con un promedio de cuatro fotografías por el anverso y reverso de las obras.

De este modo es que el presente producto logró cumplirse a cabalidad con las alrededor de 500 fotografías realizadas con cámara profesional y sistema de iluminación adecuado que entregaron imágenes en la más alta calidad. Estas fueron revisadas y anexadas finalmente tanto al catálogo como a la biografía y página web del artista. Junto con ello, se fotografiaron objetos que pertenecieron al artista, los cuales podrían ayudar a conocer más la personalidad del pintor o bien podían ser de relevancia para la investigación como, por ejemplo, las medallas obtenidas como premio en los concursos de arte que participó. A continuación, se presentan algunas fotos profesionales sobre objetos y fotografías que pertenecen a diferentes ramas de la familia Aspillaga Sotomayor realizadas durante el trabajo:

\section{Objetivo específico No3: Difundir la figura y obra de Manuel Aspillaga Valenzuela}

\section{Sitio web}

Luego de la concreción de los objetivos anteriores y su respectiva corrección, durante los meses de noviembre y diciembre se construyó el sitio web, www.manuelaspillaga.com, que contiene la biografía del artista, su análisis pictórico, contexto y la totalidad de sus obras con el fin de ser difundidas. Para esto, primeramente se redactó un resumen de la investigación desarrollada para ser anexada al sitio. Es necesario agregar que para la elaboración del sitio se recurrió al uso de las fotografías tomadas por las mismas autoras del proyecto, sobre las pinturas que forman actualmente parte del patrimonio común. Estas fotografías cuentan con la autorización de publicación por parte de la familia, ya que fueron ellos mismos quienes nos ayudaron a la recolección y posterior reproducción fotográfica. No se anexa una autorización escrita debido a la petición de Gonzalo Aspillaga, quien pidió que por edad de los dueños no se les pidiera firma.

Parte del proceso fue la compra de los derechos de página web y el pago de esta para que se encuentre en vigencia durante el periodo de un año (pudiendo luego la familia decidir si continúa manteniéndolo o no, o bien, ser parte de un nuevo proyecto a desarrollar por las autoras en una etapa posterior a la titulación). El sitio cuenta con la herramienta Google Analytics la cual medirá el número de visitas durante el periodo mencionado, por lo que el logro de la meta propuesta será evaluado en el transcurso de dicho periodo. En este sentido, el sitio se encuentra actualmente disponible con el fin de comenzar a difundir al personaje en www.manuelaspillaga.com 


\section{Publicación online}

Del mismo modo que en el producto anterior, una vez corregido el material, durante los meses de noviembre y diciembre, se ordenó el mismo a partir de un documento digital diseñado que contuviera un resumen de la información (considerando las fichas abiertas al público, no las familiares), con el fin de ser difundido a través de medios digitales. Contiene una breve historia del pintor y su contexto. Cada obra es acompañada por información básica, cómo técnica, año de creación, medidas y título. Dicho documento se encuentra actualmente en la página web, pudiendo descargarse en formato pdf. (www.manuelaspillaga.com/catálogo-digital). Del mismo modo que lo anterior, la meta propuesta está pensada para el periodo de un año por lo que la evaluación de dicho objetivo será medida en el tiempo estipulado.

En paralelo a lo anterior, se elaboró un documento personalizado para la familia donde se presentan las fichas completas de las pinturas además de la investigación. De esta manera, todos los participantes del proyecto tendrán la recopilación del trabajo realizado.

\section{Discusión}

\section{Biografía del pintor Manuel Aspillaga Valenzuela}

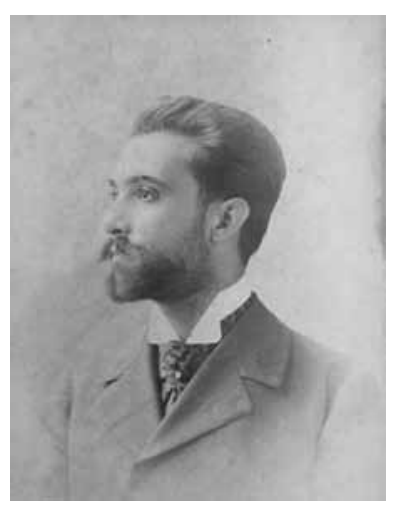

Figura 1. Retrato del pintor Manuel Aspillaga. Fuente: colección familia Aspillaga.

\section{La familia del artista}

Manuel Aspillaga nació en Santiago, el 25 de septiembre de 1870. Hijo del abogado Pedro Nolasco Aspillaga Santander (1837-s/f) y Elisa Valenzuela Castillo (1867-1949) se le bautizó con el nombre de su tío paterno y de sus abuelos, Manuel José Aspillaga Velasco y Manuel Valenzuela Castillo (1822-1889), ambos personajes que participaron activamente 
de la política del periodo. La familia aumentó cuando en 1872 nació su hermano Pedro Nolasco. Este único hermano murió soltero y sin descendencia. Hubo una tercera hermana, pero murió cuando tenía aproximadamente 10 años².

Manuel realizó sus estudios en el Colegio jesuita San Ignacio de Alonso de Ovalle ${ }^{3}$, para luego ingresar a la carrera de Derecho ${ }^{4}$ en la Universidad de Chile, de la cual egresaría el año 1893.

Abogado de profesión, fue durante su vida aficionado a la pintura, la botánica y la música, disciplina de la que participó con el aprendizaje del violín. Luego de la muerte de su padre, como hijo mayor, debió hacerse cargo de los numerosos campos de la familia, en Santiago, Pichidegua, Lolol y Tanumé5.

Durante su periodo de formación, Aspillaga vivió en la Alameda de las Delicias $\mathrm{N}^{\circ} 281$, vereda norte, frente a la calle de la Maestranza, hoy Portugal. Vivía junto a su madre viuda, Elisa Valenzuela, y a su hermano que, al igual que Manuel, había ingresado a estudiar Derecho, carrera de la que se tituló en 1895.

La familia Aspillaga Valenzuela vivía principalmente de la agricultura que producían sus fundos, entre ellos el de San Gregorio ubicado a las afueras de Santiago ${ }^{6}$, el de Pichidegua, que, si bien en un inicio servía como lugar de parada de animales y carros en el paso hacia el fundo de Callihue, con el paso del tiempo fue complementándose hasta ser una gran propiedad que heredaron los hermanos Aspillaga Valenzuela. Por otro lado, poseían el campo de Caillihue con casa en Lolol, pero que fue vendido en 1893 a Críspulo Mujica, por lo que no se mantuvo entre los descendientes Aspillaga. Por último, formaba parte de las pertenencias familiares una gran hacienda, herencia de la abuela paterna de la familia apellidada Santander, ubicada en las proximidades de Pichilemu, llamada Tanumé.

\section{Los Aspillaga Sotomayor}

Manuel Aspillaga se casó en 1895 con Raquel Sotomayor Baeza, nieta del ministro de Guerra en Campaña durante la Guerra del Pacífico: Rafael Sotomayor. Con ella criará una familia en la que nacieron cuatro hijos: Pedro (1898-1987), Manuel (1901-1970), Carlos (1902-1967) y Augusto (1914-1969).

Se mudó entonces el artista con su familia, su madre y su hermano (quien se preocupaba de los cuidados de doña Elisa Valenzuela) a una casa de dos pisos y tres patios en la Alameda de las Delicias Nº 1929.

Una personalidad de amplia cultura: amante de las artes, las letras y las ciencias naturales Manuel Aspillaga Valenzuela fue un hombre serio, estricto en la educación, austero, ordenado casi al extremo y muy inteligente ${ }^{7}$ : una persona completa que puso su pasión en diversos ámbitos que enriquecían su figura. Entre ellos se cuenta la botánica, descubriendo incluso un cactus que lleva su nombre; el arte, donde llegó a ser un gran promotor de las obras de sus contemporáneos; la música; la literatura; y el derecho, carrera que prácticamente no ejerció, pero a la que dedicó años de estudio en un periodo en que esta se constituía como una profesión tradicional que exigía la sociedad chilena.

Siempre muy cercano a la iglesia, al igual que su mujer Raquel, se preocupó durante toda su vida de adorar a Dios a través de los oratorios que poseía en cada casa y que eran complementados en algunos casos con el Santísimo, en aquellos momentos en que la fa- 
milia recibía las visitas de sacerdotes cercanos que iban a misionar la zona (en el caso de Tanumé $)^{8}$.

\section{Sus inicios en la pintura}

En el periodo en que, después de recibirse de abogado, se hace cargo de los campos de su familia, Aspillaga ingresó a formarse en las artes dedicándose preferentemente a la pintura al óleo de paisajes y marinas, siendo alumno desde 1890 del pintor Enrique Swinburn (1859-1929) $)^{9}$. Es aquí cuando el abogado santiaguino comenzará a relacionarse con destacados artistas chilenos con los que pintará en numerosas ocasiones como Onofre Jarpa, Enrique Swinburn y Thomas Somerscales, formando parte del grupo de discípulos de este último ${ }^{10}$.

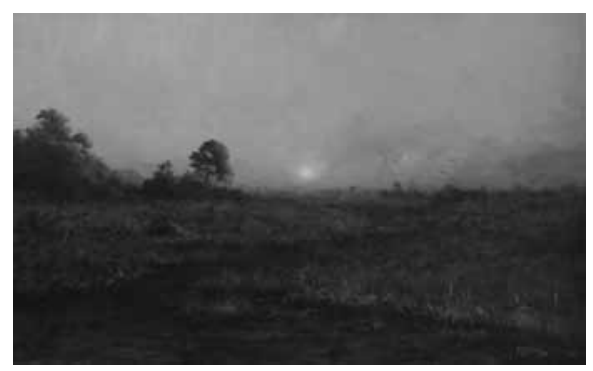

Figura 2. Obra del pintor Manuel Aspillaga. Puesta de sol en el campo óleo sobre tela $68,5 \mathrm{~cm} \times 109,5$ $\mathrm{cm}$. Fuente: colección familia Aspillaga.

Premios y reconocimientos artísticos: presencia de Manuel Aspillaga en certámenes y exposiciones de su época

1895 Mención Honrosa, sección de Pintura de la Exposición Nacional Artística. Salón de 1895. Santiago, Chile.

1896 Medalla de Segunda Clase en Pintura, sección Pintura, Acuarela y Dibujo de la Exposición Nacional Artística, Santiago, Chile.

1906 Primera Medalla en Pintura, Salón de 1906, Santiago, Chile.

1907 Premio de Paisaje del Certamen Arturo M. Edwards, Santiago, Chile.

1910 Tercera Medalla en Pintura. Exposición Internacional de Santiago. Santiago, Chile.

\section{Tanumé: refugio y creación}

Hacia el año 1913, Manuel Aspillaga finaliza la reconstrucción de su casa en Tanumé (iniciada hacia el año 1906), reemplazando la antigua casa colonial de mediados del siglo XIX por una construcción de estilo egipcio. En remembranza del palacio de la reina-faraón Hatshepsut, se incorporaron grandes columnas rematadas en capiteles con flores de loto y 
una puerta trapezoidal antecedida por dos esfinges que hizo el escultor catalán Joan Foliá Prades en 1914, junto con las columnas y algunas jarras que decoraban el recinto. La idea de construir una casa de este estilo exótico podría haber surgido del interés de Manuel Aspillaga por Egipto y su cultura, como se podía comprobar con su numerosa colección de literatura sobre dicho país ${ }^{11}$; mundo cuya fascinación alcanzó también a artistas y coleccionistas del periodo -incluso algunos chilenos, como la escultora Rebeca Matte y posteriormente el arqueólogo y coleccionista Fernando Márquez de la Plata.

Tanumé era el campo más querido y, como tal, se quiso hacer en él la mejor casa, manteniendo el interior como lo tenía su padre. De este modo, los descendientes del artista comenzaron a acudir a la gran casona familiar que se encontraba en la parte baja, junto al mar.

La casa frente al mar era una enorme construcción de pino oregón cubierta de tejas de latón y con techo plano, financiada con los ingresos de otros que permitieron transformar la antigua casona en esta exótica mansión a la que se llegaba en un tren que los transportaba desde Santiago a Alcones, donde debían subir a una carreta que los conducía hasta la casa $^{12}$, en un recorrido de cerca de cinco horas desde la capital.

El campo fue escenario de numerosas tertulias de artistas que acostumbraron a visitar a Aspillaga con el fin de pintar en sus maravillosos paisajes y compartir tardes estivales en compañía de la familia. Entre ellos, se cuenta a Onofre Jarpa (padrino de su hijo Carlos) y Enrique y Carlos Swinburn, grandes amigos del artista. Por su parte, pese a que existía cierta relación con el también artista chileno Luis Strozzi, este no era invitado en los paseos puesto que su estilo difería en el orden del resto del grupo, quienes llegaron a considerarlo muy desordenado en su arte ${ }^{13}$.

\section{Muerte del artista}

Mientras se realizaba la Exposición del Cincuentenario en el Museo de Bellas Artes, el lunes 10 de noviembre de 1930, a los 60 años, murió Manuel Aspillaga en su casa de Santiago tras una breve enfermedad ${ }^{14}$. La familia dio aviso de su fallecimiento, publicando la noticia de defunción en El Diario Ilustrado y en El Mercurio de Santiago, informando que se realizaría un funeral privado en el Cementerio General donde sería enterrado el artista en un mausoleo familiar del recinto.

\section{Resultados}

A partir de la siguiente matriz de marco lógico establecida al inicio del proyecto, es posible apreciar los siguientes resultados según producto establecido. 
Objetivo específico 1: $\quad$ Investigar la vida y obra pictórica de Manuel Aspillaga.

Producto 1:

Indicador:

Resultado esperado:

Medio de verificación:

Resultado:

Conclusión:

Producto 2:

Indicador:

Resultado esperado:

Medio de verificación:

Resultado:

Conclusión:

Objetivo específico 2:

Producto 1:

Indicador:

Resultado esperado:

Medio de verificación:

Resultado:

Conclusión:

Producto 2:

Indicador:

Resultado esperado:

Medio de verificación:

Resultado:

Conclusión:

Objetivo específico 3:

Producto 1:

Indicador:

\section{Biografía.}

Fuentes bibliográficas.

Entrevistas con familia.

Mínimo diez fuentes de información (bibliografía, entrevistas, etc).

Número de referencias bibliográficas encontradas.

Cantidad de familiares entrevistados.

Se hizo uso de 18 fuentes bibliográficas, diez entrevistas familiares y cuatro fuentes de información detalladas en la bibliografía.

Logrado

Análisis crítico de la obra.

Cuadros analizados.

Mínimo 30 obras pictóricas.

Análisis pictórico de las obras fotografiadas en terreno.

Se analizaron 50 obras, su iconografia y técnica pictórica.

Logrado

Identificar y catalogar las obras del artista.

Catalogación de las obras.

Cantidad de fichas de cuadros.

Mínimo 30 cuadros para fichar.

Comprobación en terreno de la existencia.

Se realizaron 50 fichas de catalogación.

Logrado

Fotografías profesionales.

Cuadros analizados.

Mínimo 30 obras para fotografiar.

Cantidad de cuadros.

Se fotografiaron 50 obras.

Logrado
Difundir la figura y obra de Manuel Aspillaga

Sitio web

Cantidad de visitas al sitio web después de la promoción y durante un año. 
Resultado esperado:

Medio de verificación:

Resultado:

Conclusión:

Producto 2:

Indicador:

Resultado esperado:

Medio de verificación:

Resultado:

Conclusión:
$\mathrm{Al}$ menos 150 visitas el día de la promoción en medios.

Conteo de vistas por medio de Google Analitycs.

Se evaluará después del lanzamiento y aparición en medio $E l$ Mercurio.

Por verificar durante el año.

Catálogo digital público con la vida y sus obras.

Cantidad de descargas del pdf después de la promoción y durante un año.

Al menos 150 visitas el día de la promoción en medios y 200 descargas durante el año.

Conteo de descargas por medio de Google Analitycs.

Se evaluará después del lanzamiento y aparición en medio $E l$ Mercurio.

Por verificar durante el año.

Por lo tanto, según lo establecido con anterioridad es posible afirmar que se lograron los objetivos propuestos para el presente proyecto. De todos modos, es necesario aclarar que el tercer objetivo específico y sus productos asociados fueron realizados como ha sido descrito, pero serán evaluados por el tiempo mencionado de un año (concretándose esto en diciembre del año 2019).

\section{Conclusiones}

Una vez concluido el proyecto consideramos que este es un aporte inédito a la historia del arte nacional. Junto con construir una parte de la historia de arte que no era conocida y dar valor a un artista y su obra, pone a disposición de la comunidad dicha información de valor patrimonial. Cabe destacar, además, que gracias a la incorporación de la tecnología y el diseño se logró que la información sobre la vida y obra siga viva en una plataforma digital y que, a través de ella, la comunidad pueda involucrarse colaborativamente pudiendo entregar nuevos antecedentes o referencias para enriquecer el trabajo constantemente. Es importante resaltar en el proyecto el trabajo interdisciplinario como una manera de lograr innovar a la hora de potenciar el alcance de la difusión para darlo a conocer. Esto basándonos en la premisa que nos motiva "no se cuida ni valora lo que no se conoce". Por otra parte, esta metodología de trabajo es una manera contemporánea y atractiva aportada desde experiencia de ejecutar este proyecto y puede ser replicable en otros proyectos de investigación patrimonial, acercando así este tipo de contenido a las personas y a su vez resguardarlo. 


\section{Notas}

1. Museo Nacional de Bellas Artes. Manuel Aspillaga. Artistas Visuales Chilenos. Disponible en https://www.artistasvisualeschilenos.cl/658/w3-article-39924.html. Accedido en 6/6/2019.

2. Entrevista realizada por Hernán Rodríguez Villegas a Sergio Jarpa Yáñez, viudo de Inés Aspillaga Salas, nieta de Manuel Aspillaga Valenzuela, año 2018, Vitacura, Santiago, Chile. 3. Información entregada por la familia, año 2018.

4. Entrevista realizada por Hernán Rodríguez Villegas a Sergio Jarpa Yáñez, Op. Cit.

5. Entrevista realizada por Hernán Rodríguez Villegas a Sergio Jarpa Yáñez, Op. Cit.

6. La propiedad se vendió alrededor de 1956-1958 dando origen a la actual población San Gregorio en la comuna de la Granja, en las afueras de Santiago.

7. Entrevista a Carmen María Barros Aspillaga, Op. Cit.

8. Entrevista a Gonzalo Aspillaga Herrera, Op.Cit.

9. Entrevista realizada por Hernán Rodríguez Villegas a Sergio Jarpa Yáñez, Op. Cit.

10. Museo Nacional de Bellas Artes, Artistas Visuales Chilenos, Manuel Aspillaga en http:// www.artistasvisualeschilenos.cl/658/w3-article-39924.html (Consultado el 22/04/2018).

11. Entrevista a Julia Rodríguez Ortúzar, viuda de Fernando Aspillaga Salas, nieto de Manuel Aspillaga Valenzuela, 8 de octubre de 2018, Providencia, Santiago, Chile.

12. Entrevista a Sergio Jarpa Yáñez, Op. Cit.

13. Entrevista a Julia Rodríguez Ortúzar, Op. Cit.

14. Existe otra versión entregada por Gonzalo Aspillaga que dice que el artista murió como consecuencia del impacto generado por un desastre natural que destruyó la quebrada a la que tanto tiempo le había dedicado en Tanumé. Esto le habría provocado un ataque al corazón que le impediría llegar con vida hasta Santiago, muriendo en la estación de trenes de Alcones, camino al hospital.

\section{Referencias}

Allamand, A. F. (2008). Pintura Chilena del siglo XIX. Pedro Lira. Santiago: Ediciones Origo, Santiago.

Biblioteca del Congreso Nacional (s/f). Reseña Biográfica Manuel José Aspillaga Velasco, disponible en https://www.bcn.cl/historiapolitica/resenas_parlamentarias/wiki/Manuel _Jos\%C3\%A9_Aspillaga_Velasco. Consultado el 16 de octubre de 2018.

Bindis, R. (2006). Pintura Chilena doscientos años. Santiago: Ediciones Origo, Primera Edición.

Cruz, I. (2004). “El paisaje chileno en los pintores viajeros del romanticismo” en Patrimonio Cultural $N^{\circ} 33$.

Diario El Ferrocarril, 16 de noviembre de 1896, Santiago, Chile.

El Diario Ilustrado, 12 de noviembre de 1930, Santiago, Chile.

Gombrich, E. (1950). La historia del arte. Nueva York: Editorial Phaidon, 16th edición, 1997. Honour, H. (2004). El romanticismo. Madrid: Alianza Editorial. 
Museo Nacional de Bellas Artes, Artistas Visuales Chilenos, Giovanni Mochi, disponible en http://www.artistasvisualeschilenos.cl/658/w3-article-40026.html. Consultado el 26 de noviembre de 2018.

Museo Nacional de Bellas Artes, Artistas Visuales Chilenos, Manuel Aspillaga, disponible en http://www.artistasvisualeschilenos.cl/658/w3-article-39924.html. Consultado el 22 de abril de 2018.

Museo Nacional de Bellas Artes (archivo), Carpeta de Manuel Aspillaga Valenzuela. Consultada el 4 de junio de 2018.

Museo Nacional de Bellas Artes, Catálogo de la Exposición Nacional Artística del Salón de 1908, Sociedad Imprenta y Litografía Universo, Santiago de Chile, 1908, disponible en http://www.mnba.cl/617/articles-9370_archivo_01.pdf. Consultado el 18 de abril de 2018.

Museo Nacional de Bellas Artes, Catálogo Oficial ilustrado de la Exposición internacional de Bellas Artes, Imprenta Barcelona, Santiago de Chile, 1910, disponoble en http://www. mnba.cl/617/articles-9350_archivo_01.pdf. Consultado el 10 de mayo de 2018).

Piaget, J. (1979). "La epistemología de las relaciones interdisciplinarias”, en: L. Apostel, G. Bergerr, A. Bringgs y G. Michaud, Interdisciplinariedad. Problemas de la enseñanza y de la investigación en la Universidades. México: Asociación Nacional de Universidades e Institutos de Enseñanza Superior.

Quiroga, S. y Villegas, L. (2015). Antonio Smith. Temuco: Universidad Católica de Temuco. Reynols, D. M. (1996). Introducción a la historia del arte, el siglo XIX. Barcelona: Editorial Gustavo Gili, 4th Edición.

Rodríguez, G. C. y Vergara, J. (2011). “Representando la “Copia Feliz del Edén”. Rugendas: Paisaje e Identidad nacional en Chile, Siglo XIX”, en Revista de Historia Social y de las Mentalidades, Volumen 15, N. ${ }^{\circ} 2$.

Romera, A. (1951). Historia de la pintura chilena. Santiago: Editorial del Pacífico, 1th Edición. Valenzuela, J. (1923). Álbum zona central de Chile. Informaciones agrícolas, 1923, disponible en http://www.memoriachilena.cl/archivos2/pdfs/MC0007868.pdf. Consultado el 22 de abril de 2018.

\section{Entrevistas}

Aspillaga Barros, Carmen Gloria, nieta de Manuel Aspillaga Valenzuela, 10 de septiembre 2018, Las Condes, Santiago, Chile.

Aspillaga Herrera, Gonzalo, nieto de Manuel Aspillaga Valenzuela, 29 de agosto 2018, Vitacura, Santiago, Chile.

Barros Aspillaga, Carmen María, nieta de Manuel Aspillaga Valenzuela, 1 de octubre 2018, Las Condes, Santiago, Chile.

Barros Aspillaga, José Miguel, nieto de Manuel Aspillaga Valenzuela, 4 de octubre 2018, Las Condes, Santiago, Chile.

Barros Bezanilla, Ana María, viuda de Pedro Aspillaga Salas, nieto de Manuel Aspillaga Valenzuela, 4 de octubre 2018, Las Condes, Santiago, Chile. 
Gazitúa Costabal, Irene, viuda de Jaime Aspillaga Barros, nieto de Manuel Aspillaga Valenzuela, 30 de agosto 2018, Vitacura, Santiago, Chile

Jarpa Yáñez, Sergio, viudo de Inés Aspillaga Salas, nieta de Manuel Aspillaga Valenzuela, 10 de septiembre 2018, Vitacura, Santiago, Chile.

Rodríguez Ortúzar, Julia, viuda de Fernando Aspillaga Salas, nieto de Manuel Aspillaga Valenzuela, 8 de octubre 2018, Providencia, Santiago, Chile.

\title{
Otros
}

Entrevista realizada por Hernán Rodríguez Villegas a Sergio Jarpa Yáñez, viudo de Inés Aspillaga Salas, nieta de Manuel Aspillaga Valenzuela, año 2018, Vitacura, Santiago, Chile. Información entregada por Hernán Rodríguez Villegas, año 2018.

Información entregada por Isabel Margarita Finlay viuda de Cristián Aspillaga Herrera, nieto de Manuel Aspillaga Valenzuela, el día 8 de octubre del 2018.

Información entregada por la familia, año 2018.

\begin{abstract}
This article describes a research project conducted in 2018, through interdisciplinary work between design, history, and the use of technological resources, to rescue, register and disseminating heritage.

The main objective was to give value to the life and work of the unknown Chilean painter of the late 19th Century Manuel Aspillaga Valenzuela (1870-1930), and disseminate the results through digital resources on a free access online platform.

In terms of content the project succeeds in placing the artist and his work within the national historic context landmark. Along with the above, the project seeks to provide access to the entire community to these unknown heritage through technology and design.

It is important, in addition, to describe the methodology of interdisciplinary work used for the realization of the objectives. The methodology enabled to achieve a systematic and deep research whose success is based on enhancing each of the disciplines involved in the investigation, execution, analysis and creation of pieces.

The Project was developed in three phases, Investigate the life and pictorial work of Manuel Aspillaga; identifying, registering and cataloguing the artist's work; and, finally, to divulge the figure and work of Manuel Aspillaga Valenzuela. This study took place in Santiago, Chile during the course of a year.
\end{abstract}

Keywords: design - interdiscipline - heritage - art - technology.

Resumo: Este artigo descreve como, através de um trabalho interdisciplinar entre design, história e uso de recursos tecnológicos, um projeto de pesquisa, resgate, registro e disseminação é desenvolvido em 2018. 
O objetivo principal era valorizar a vida e a obra do pintor chileno desconhecido do final do século XIX, Manuel Aspillaga Valenzuela (1870-1930), e depois divulgar os resultados por meio de recursos digitais localizados em uma plataforma de acesso on-line gratuito. Em termos de conteúdo, o projeto consegue colocar o artista e sua obra no contexto histórico nacional. Junto com o exposto, o projeto busca dar acesso a toda a comunidade a esses trabalhos de herança desconhecidos por meio de tecnologia e design. Também é relevante descrever a metodologia de trabalho interdisciplinar usada para alcançar os objetivos. Com essa metodologia, foi realizado um trabalho ordenado e profundo, cujo sucesso se baseou no fortalecimento de cada uma das disciplinas envolvidas na pesquisa, execução, análise e criação de peças. O projeto foi desenvolvido em três etapas, investigando a vida e obra pictórica de Manuel Aspillaga; identificar, registrar e catalogar as obras do artista; e, finalmente, espalhar a figura e o trabalho de Manuel Aspillaga Valenzuela.

Esta investigação foi realizada na cidade de Santiago do Chile dentro de um ano.

Palavras chave: design - interdisciplinar - património - arte - tecnologia.

[Las traducciones de los abstracts fueron supervisadas por el autor de cada artículo] 